\title{
Capsular Contracture Rates in Augmentation Mammaplasty: Comparison of Round vs Anatomical Breast Implants
}

\author{
Sangdal Lee, M.D., Ph.D., Min Kuk Kim, M.D., Seunggeun Lee, M.D., Ph.D., Woosang Ryu, M.D., \\ Seunghee Back, M.D. \\ MD Clinic, Breast Center, Seoul, Korea
}

Purpose: Capsular contracture is a major cause of poor postoperative satisfaction in augmentation mammaplasty. It is unclear whether round or anatomical textured breast implants lead to differences in the rates of capsular contracture. Accordingly, the objective of this study was to compare capsular contracture rates between round and anatomical textured breast implants. Methods: A total of 1,282 cases (2,564 breasts) of augmentation mammaplasty using textured type implants, performed at MD clinic between January 2012 and December 2015 , were retrospectively reviewed. A total of 703 cases used round textured implants while 579 used anatomical textured implants. The median follow-up period for the round textured implant group was 9 months, and 7 months for the anatomical textured group. Results: The overall capsular contracture rates in the first year was $2.0 \%$ in the round textured group and $1.5 \%$ in the anatomical textured group ( $p=0.609)$. Capsular contracture rates in the first year in those who underwent primary surgery were $1.6 \%$ in the round textured group and $1.3 \%$ in the anatomical textured group $(p=0.187)$. The rates in those who underwent revision surgery were $5.2 \%$ in the round textured group and $2.6 \%$ in the anatomical textured group $(p=0.178)$. Conclusion: Although the risk for capsular contracture in the anatomical textured groups appeared to be slightly lower than in the round textured groups, the difference was not significant. Further studies with longer follow-up periods are needed to assess potential differences.

Key Words: Breast implants, Implant capsular contracture, Mammaplasty, Silicone ge/s

\section{INTRODUCTION}

Augmentation mammaplasty is procedure used to increase breast size by inserting implants under the breast parenchyma or pectoralis muscles. The most common complication after surgery is capsular contracture due to over-forming capsule around the implants [1]. Capsular contracture is a major cause of poor postoperative satisfaction because it compresses the implant and causes distortion in shape, hardening, and asymmetry. It is classified as grades 1 to 4 according to the classification described by Baker, a subjective classification system that is based on clinical findings by the physician. All patients undergoing breast augmentation have a Grade I capsule which is soft, supple, and non-palpable. Grade II describes breasts that are somewhat firm but appears normal. Grade III breasts are firm and appears abnormal, and Grade IV breasts are hard, painful to the touch, and appears ab-

Correspondence: Sangdal Lee, M.D., Ph.D.

MD Clinic, Breast Center, 814 Seolleung-ro, Gangnam-gu, Seoul 06014, Korea Tel: +82-2-542-0081, Fax: +82-2-542-4443, E-mail: dahl65@hanmail.net Received: Nov 21, 2018 Revised: Mar 26, 2019 Accepted: May 22, 2019 normal. Grades III and IV breasts are symptomatic and clinically significant. Capsular contracture can occur in one or both sides at any point after mammaplasty, and most cases are likely to occur within the first 6 months after surgery. The incidence rates of capsular contracture have been reported to vary from $2.8 \%$ to $20.4 \%$ [2-6]. The main reason for such variation in incidence rates is that studies have been performed without sufficient preventive or standardized criteria for various factors that can lead to capsular contracture. Thus, it is necessary to compare incidence rates by excluding as many such factors as possible.

There are many reasons for the occurrence of capsular contracture and, because there are large differences according to implant characteristics, many improved products have been introduced. For example, textured type implants have demonstrated relatively low capsular contracture rates compared with smooth type implants [7,8]. Among the textured types, round and anatomical implants are recently being widely used recently. However, it is currently unclear whether these types of textured breast implants lead to differences in the rates of capsular contracture. Therefore, the objective of this study was to compare 
capsular contracture rates between round and anatomical textured implants.

\section{METHODS}

Between January 2012 and December 2015, a total of 1,282 (2,564 breasts) cases of augmentation mammaplasty using textured type implants, without complication of hematoma, were performed at MD clinic were retrospectively reviewed (IRB No. 2020-0417-002). A total of 703 cases involved the round textured type while 579 involved the anatomical textured type. Primary surgery was performed in 1,107 cases, while revision surgery was performed in 175 (Table 1). Three types of incision were made: axillary, peri-areolar, and inframamma-

Table 1. Clinical characteristics of cases

\begin{tabular}{|c|c|c|c|}
\hline Characteristic & $\begin{array}{c}\text { Round-type } \\
\text { No. (\%) }\end{array}$ & $\begin{array}{c}\text { Anatomical-type } \\
\text { No. (\%) }\end{array}$ & $p$-value \\
\hline Age (yr) & & & $<0.001$ \\
\hline less than 30 & $298(42.4)$ & $197(34.0)$ & \\
\hline $30-39$ & $306(43.5)$ & $233(40.2)$ & \\
\hline $40-49$ & $81(11.5)$ & $125(21.6)$ & \\
\hline 50 or more & $18(2.6)$ & $24(4.2)$ & \\
\hline Total & $703(100.0)$ & $579(100.0)$ & \\
\hline Mean \pm SD (range) & $31.8 \pm 7.2(18-57)$ & $33.8 \pm 8.0(19-62)$ & 0.002 \\
\hline Location of incision & & & $<0.001$ \\
\hline axillary & $422(60.0)$ & $250(43.2)$ & \\
\hline periareolar & $168(23.9)$ & $153(26.4)$ & \\
\hline inframammary & $113(16.1)$ & $176(30.4)$ & \\
\hline \multicolumn{4}{|l|}{ Volume of implants (cc) } \\
\hline Right $^{*}$ & $272.7(190-401)$ & $304.8(160-375)$ & 0.004 \\
\hline less than 200 & $9(1.3)$ & $4(0.7)$ & \\
\hline $200-249$ & $142(20.2)$ & $14(2.4)$ & \\
\hline $250-299$ & $378(53.8)$ & $264(45.6)$ & \\
\hline $300-349$ & $163(23.2)$ & $249(43.0)$ & \\
\hline 350 or more & $11(1.6)$ & $48(8.3)$ & \\
\hline Left $^{*}$ & $264.9(180-401)$ & $289.0(140-375)$ & 0.104 \\
\hline less than 200 & $19(2.7)$ & $8(1.4)$ & \\
\hline $200-249$ & $185(26.3)$ & $49(8.5)$ & \\
\hline $250-299$ & $375(53.3)$ & $294(50.8)$ & \\
\hline $300-349$ & $118(16.8)$ & $202(34.9)$ & \\
\hline 350 or more & $6(0.9)$ & $26(4.5)$ & \\
\hline Type of operation & & & 0.410 \\
\hline $\begin{array}{l}\text { Primary augmentation } \\
(n=1,107)\end{array}$ & $602(85.6)$ & $505(87.2)$ & \\
\hline $\begin{array}{l}\text { Augmentation revision } \\
(n=175)\end{array}$ & $101(14.4)$ & $74(12.8)$ & \\
\hline $\begin{array}{l}\text { Median follow-up period } \\
(\mathrm{mo})^{+}\end{array}$ & $9.0(1-32)$ & $7.0(1-30)$ & \\
\hline
\end{tabular}

${ }^{*}$ Mean (range); ${ }^{\dagger}$ No. (range). ry. All cases were performed using the subpectoral dual plane technique under general anesthesia. The endoscopic approach was used in all cases of axillary incisions. For revision cases, subpectoral insertion was performed after removing the capsule using a peri-areolar or inframammary incision. All patients were treated with antibiotics (cefradine $1.0 \mathrm{~g}$ ) in the exfoliated space during surgery. Oral antibiotic (cefradine $1.5 \mathrm{~g}$ ) was then administered for five days after surgery. In all cases, a closed-suction drain was inserted in both breast pockets to prevent hematoma formation and drainage was removed 2-4 days later depending on case status. The diagnosis of capsular contracture was assessed by physical examination at 3 and 12 months after the operation by the surgeon who performed the surgery; follow-up was performed annually thereafter. Diagnosis was based on Baker classification grades III and IV. Capsular contracture in one or both breasts was designated as 1 case.

Statistical analysis was performed using the independent-sample t-test, chi-squared test, and Fisher's exact test to compare data between the two groups. In terms of capsular contracture rate, survival rates were calculated and compared using the Kaplan-Meier method and log-rank test. All statistical analyses were performed using R version 3.2.5 (R Foundation for Statistical Computing, Vienna, Austria [http://www.R-project.org]); $p<0.05$ was considered to be statistically significant.

\section{RESULTS}

The age of the subjects ranged from 18 to 62 years. The average age of subjects in the round textured group was 31.8 years and 33.8 years in the anatomical textured group $(p=0.002)$ (Table 1). In terms of implant size, average sizes were $272.7 \mathrm{cc}$ for the right side and $264.9 \mathrm{cc}$ for the left side in the round textured group, and $304.8 \mathrm{cc}$ for the right side and $289.0 \mathrm{cc}$ for the left side in the anatomical textured group. The median follow-up period for the round type group was 9 months, and 7 months for the anatomical type group.

The overall capsular contracture rates in the first year was 2.0\% in the round textured implant group and $1.5 \%$ in the anatomical textured implant group; the difference between the two groups, however, was not statistically significant $(p=0.609)$ (Table 2, Figure 1). The capsular contracture rates in the first year in those who underwent primary surgery was $1.6 \%$ in the round textured group and $1.3 \%$ in the 
Table 2. Kaplan-Meier estimated one-year capsular contracture rates comparing round textured and anatomical textured implants in patients with primary and revision breast augmentation

\begin{tabular}{|c|c|c|c|}
\hline Type of operation (\%) & $\begin{array}{l}\text { Round type } \\
\text { Rate }(95 \% \mathrm{Cl})\end{array}$ & $\begin{array}{l}\text { Anatomical type } \\
\text { Rate }(95 \% \mathrm{Cl})\end{array}$ & $p$-value \\
\hline Primary augmentation & & & 0.187 \\
\hline 3 months & $0.8(0.0-1.5)$ & $0.3(0.0-0.7)$ & \\
\hline 1 year & $1.6(0.4-2.7)$ & $1.3(0.0-2.8)$ & \\
\hline Augmentation revision & & & 0.178 \\
\hline 3 months & $5.2(0.0-10.2)$ & 0.0 & \\
\hline 1 year & $5.2(0.0-10.2)$ & $2.6(0.0-7.4)$ & \\
\hline $\begin{array}{l}\text { Overall capsular contracture } \\
\text { rate }\end{array}$ & & & 0.609 \\
\hline 3 months & $1.3(0.4-2.2)$ & $0.2(0.0-0.6)$ & \\
\hline 1 year & $2.0(0.8-3.2)$ & $1.5(0.0-2.9)$ & \\
\hline
\end{tabular}

$\mathrm{Cl}=$ confidence interval.

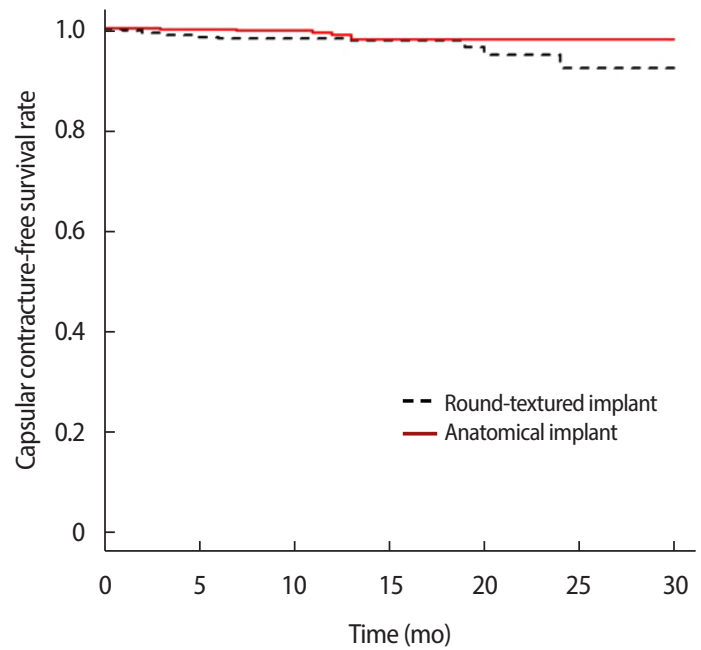

Figure 1. Capsular contracture-free survival curves according to the Kaplan-Meier method comparing round textured and anatomical textured breast implants $(p=0.130)$.

anatomical textured group, and the rates in those who underwent revision surgery was $5.2 \%$ in the round textured group and $2.6 \%$ in the anatomical textured group. There was no statistically significant difference in the incidence rates of capsular contracture between the two implant groups according to surgery type (primary surgery, $p=0.187$; revision surgery, $p=0.178$ ) (Figure 2 ).

In comparing of capsular contracture rates according to incision type between the two groups, the rate was $1.6 \%$ in the round textured group and $0.5 \%$ in anatomical textured group for those in whom an axillary incision was used $(p=0.202)$. In those in whom a periareolar incision was used, the rates were $3.1 \%$ in the round textured group and $2.3 \%$ in the anatomical textured group with no significant difference
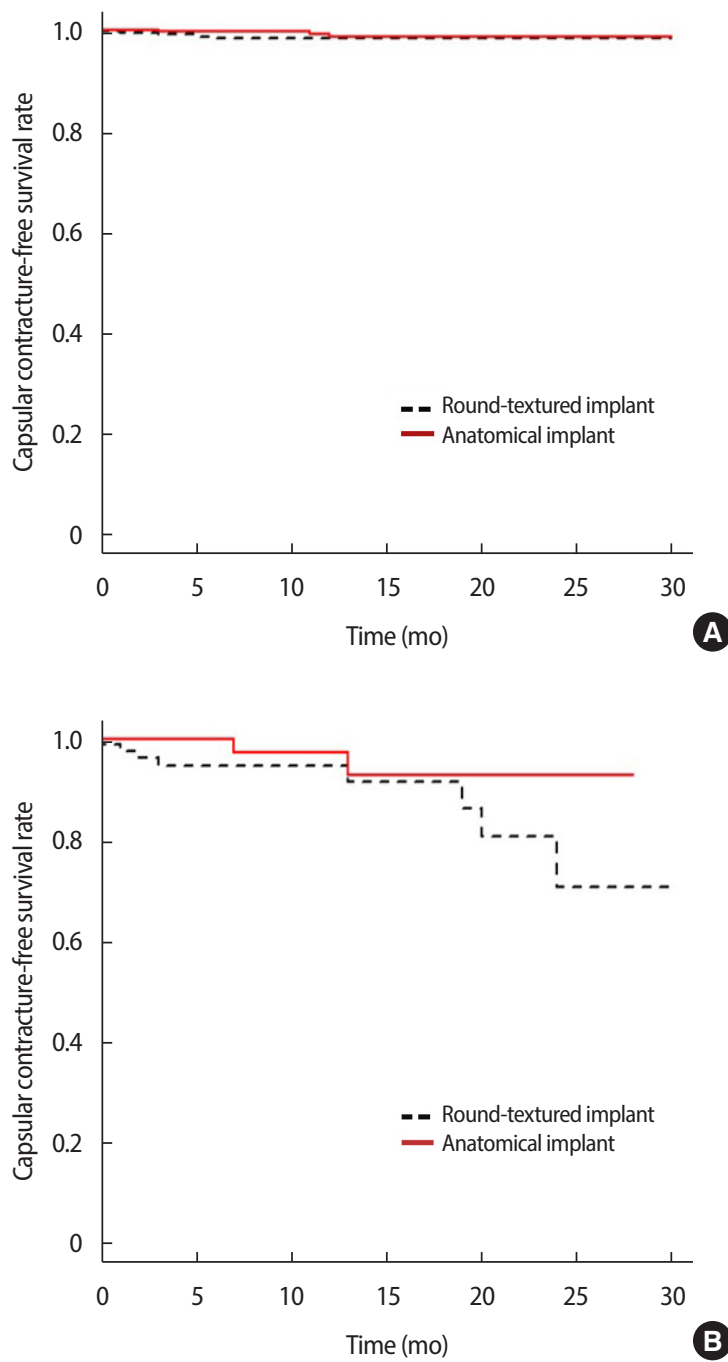

Figure 2. Capsular contracture-free survival curves comparing round textured and anatomical textured implants in primary surgery $(A)$ and revision surgery augmentation (B) ( $p=0.361, p=0.178$, respectively).

between the two groups $(p=0.798)$. However, in cases for which an inframammary incision was performed, statistical comparison could not be performed because the rates in the anatomical textured group was $2.1 \%$, while no capsular contractures were observed in the round textured group.

In univariate analysis, revisional augmentation was the only significant factor for higher rates of capsular contracture. Although the age distribution of subjects and implant sizes were statistically different between the two groups, these were not significant factors influencing differences in the rates of capsular contracture. The reason for the larger size of the right-side implants compared with the left was that, in most cases, the left breast was larger or the left chest wall was more 
protruded; these also were not significant factors in the rates of capsular contracture. After adjusting for age, type of surgery, implant size, and incision type, multivariate analysis revealed that patients who underwent revision surgery exhibited a significantly higher risk for capsular contracture than those who underwent primary augmentation ( $p=0.015$; hazard ratio 3.62 [95\% confidence interval 1.26-9.06]). This may have been related to the reason for revision surgery. Most cases requiring revision had a history of pre-existing capsular contracture due to the primary surgery. In subjects who required revision, however, there was no significant difference in the rates of capsular contracture between the round and anatomical type implants.

\section{DISCUSSION}

Breast implants using silicone gel have been continuously developed since their initial introduction by Cronin and Gerow in 1964 [9]. In 2006, cohesive silicone gel was marketed worldwide as a universal breast implant. With advances and developments in implant technology and surgical techniques, the range of choices has been expanded for both patients and physicians, ranging from smooth to textured type, with round and anatomical shapes. Anatomical type implants have made it possible to realize a more natural breast shape compared with the round type. Although the surface material used in round and anatomical type implants is essentially the same, a highly cohesive silicone gel is used to maintain the original shape in anatomical type implants, also known as "form-stable" implants. Because the silicone gel used in anatomical type implants has high cohesiveness and is full-filling, the implant is relatively firm and resistant to physical forces exerted on the capsule by contracture [10]. This means that changes in tactile sensation and breast deformity due to capsular contracture in anatomical type implants may not be clinically evident compared with those in round type implants.

In terms of lower capsular contracture rates in anatomical type implants, the high cohesive force of the internal silicone gel is a noteworthy factor. High-intensity cohesive forces may resist compression of the capsule. Due to this high cohesion, which is comparatively firm to the touch, subjects may not sense slight capsular contracture. In a 10year follow up study investigating the McGhan style 153 dual-lumen implant, McGhan, Hammond and Schmitt [11] reported rates of capsular contracture (Baker grade III/IV) as high as 51.5\%. Style 153 was a dual-lumen anatomical type implant covered by a shell similar to the Biocell type manufactured by Allergan (Irvine, CA, USA). It is filled with silicone gel with intermediate cohesiveness. This product has been discontinued due to high rupture rates resulting from a weak shell. A notable result was that high rates of implant rupture were associated with high rates of capsular contracture. Although the reason has not been clearly explained, silicone gels with low cohesiveness may not be able to resist pressure of the capsule. In addition, the shell was so easily wrinkled that the capsule lacked complete adhesion. This may have resulted in a high risk for chronic inflammation. Although rotation of round type implants can be ignored because of no change in shape, the possibility of rotation is higher than that in anatomical type implants, which increases the likelihood of adhesion failure or seroma. More specifically, in round type implants filled with silicone gel with lower cohesiveness, compared with anatomical type implants filled with higher cohesiveness, resistance to capsular compression is low, resulting in frequent wrinkling and folding of the shell. This may lead to a risk for adhesion failure. It may also increase the risk for chronic inflammation and seroma around the implant, thus increasing the risk for capsular contracture.

In addition to the type of implants, incision site, insertion location of the implants, such as submuscular or subglandular (and subfascial) pockets based on the pectoralis major muscle, inflammation, and postoperative hematoma are also important factors that can affect the incidence of capsular contracture [7,12]. Therefore, in this study, cases of implantation involving textured implants via submuscular insertion performed by a single surgeon were included to minimize the possibility of differences caused by multiple surgeons and varying surgical methods; moreover, subjects who experienced hematoma were excluded. It is generally accepted that subpectoral insertion has a lower incidence of capsular contracture than the subglandular or subfascial insertion method $[13,14]$. However, controversy regarding the difference in capsular contracture rates depending on the incision site is ongoing. In a study investigating the Natrelle 410 anatomical type implantmanufactured by Allergan Mcguire et al. [13] found that cases of peri-areolar incision exhibited higher capsular contracture rates than those involving the inframammary incision. Stutman et al. [4] reported that axillary incision had the highest capsular contracture rates, followed by periareolar and inframammary incision. Benito-Ruiz et al. [14] and Jacobson et al. [15] reported that peri-areolar in- 
cision resulted in relatively higher rates, while inframammary incision demonstrated relatively lower rates of capsular contracture, although the difference between the two was not statistically significant. In the present study, there was no significant difference in capsular contracture rates among the incision sites, although relatively higher rates were observed in cases involving peri-areolar insertion.

Previous studies have also compared capsular contracture rates of round and anatomical type implants. In a 9-year follow up study investigating implants manufactured by Mentor published in 2014, Caplin [10] reported that the incidence of capsular contracture was lower in anatomical contour profile gel (CPG) type than that in round type. Hammond et al. [16] have reported similar results regarding implants manufactured by Mentor (Santa Barbara, CA, USA). Spear and Murphy [2] reported that the Natrelle Style 410 anatomical type implant manufactured by Allergan exhibited lower capsular contracture rates than the round type; Maxwell et al. [17] reported similar results. However, smooth type implants were included in round type cases in all of these studies. It has been reported that smooth type implants exhibit higher rates of capsular contracture than the textured type $[7,8,17,18]$. Thus, smooth type implants should be excluded from round type cases to obtain an accurate comparison between round textured and anatomical textured type implants. As such, results of the present study are valuable because differences in the rates of capsular contracture between round and anatomical textured implants were investigated after excluding smooth type implants from round cases. Results of 1-year follow-up revealed that the incidence of capsular contracture was lower in the anatomical type than that in the round textured type implant in overall and incision site comparisons. The incidence rates of capsular contracture were slightly lower in the group using the anatomical type (1.5\%) than in the round type group (2.0\%) in this study. However, the difference between the two groups was not statistically significant.

This was a retrospective study and, because anatomical type implants have recently begun to be used more frequently compared with the round type, there was a difference in the median follow-up period between the two groups. Thus, the follow-up period in this study was insufficient compared with those reported in the existing literature. However, despite the short follow-up period, the duration of follow-up was similar between the two groups and the number of subjects was sufficient to enable meaningful analysis. Comparisons in this study were performed using cases involving textured type implants performed at a single center by a single surgeon. This has not been done in previous studies. Similar to other studies investigating the incidence of capsular contracture, the present study also compared and analyzed rates of capsular contracture after 1 year after the operation using the Kaplan-Meier method by summing various follow-up periods of all cases within the study period. Large scale, multicenter, prospective reports from Mentor or Allergan also used the Kaplan-Meier method to verify and perform comparative analysis of capsular contracture, re-operation frequency, and other complications, and reported risk ratios $[2,16,17]$.

The present study revealed that differences in gel cohesiveness could be a factor contributing to the lower rates of capsular contracture in anatomical type implants, although the difference in the rates of capsular contracture was not statistically significant between round type and anatomical type implants. No significant difference in the rates of capsular contracture between the two may be due to the fact that the same shell is used in both types. As a result of comparison with 1-year of follow up using the Kaplan-Meier method (Figure 1), there may be a tendency toward differences observed over time. Significant differences may be evident with longer-term follow-up, and, as such, further study is needed to clarify such possibilities.

\section{REFERENCES}

1. Handel N, Cordray T, Gutierrez J, Jensen JA. A long-term study of outcomes, complications, and patient satisfaction with breast implants. Plast Reconstr Surg 2006;117:757-67.

2. Spear SL, Murphy DK. Allergan silicone breast implant USCCSG. Natrelle round silicone breast implants: core study results at 10 years. Plast Reconstr Surg 2014;133:1354-61.

3. Blount AL, Martin MD, Lineberry KD, Kettaneh N, Alfonso DR. Capsular contracture rate in a low-risk population after primary augmentation mammaplasty. Aesthet Surg J 2013;33:516-21.

4. Stutman RL, Codner M, Mahoney A, Amei A. Comparison of breast augmentation incisions and common complications. Aesthetic Plast Surg 2012;36:1096-104.

5. Codner MA, Mejia JD, Locke MB, Mahoney A, Thiels C, Nahai FR, et al. A 15-year experience with primary breast augmentation. Plast Reconstr Surg 2011;127:1300-10. 
6. Sevin A, Sevin K, Senen D, Deren O, Adanali G, Erdogan B. Augmentation mammaplasty: retrospective analysis of 210 cases. Aesthetic Plast Surg 2006;30:651-4.

7. Stevens WG, Nahabedian MY, Calobrace MB, Harrington JL, Capizzi PJ, Cohen R, et al. Risk factor analysis for capsular contracture: a 5-year sientra study analysis using round, smooth, and textured implants for breast augmentation. Plast Reconstr Surg 2013; 132:1115-23.

8. Headon H, Kasem A, Mokbel K. Capsular contracture after breast augmentation: an update for clinical practice. Arch Plast Surg 2015; 42:532-43.

9. Cronin TD, Gerow RM. Augmentation mammaplasty: a new "natural feel" prosthesis. In translations of the third international congress of plastic surgery. Excerpta medica international congress series. No. 66. Amsterdam: Excerpta Medica; 1964.p. 41-9.

10. Caplin DA. Indications for the use of memory shape breast implants in aesthetic and reconstructive breast surgery: long-term clinical outcomes of shaped versus round silicone breast implants. Plast Reconstr Surg 2014;134:S27-37.

11. Hammond DC, Schmitt WP. Long-term outcomes with the McGhan style 153 dual-lumen breast implant: implications for future implant design. J Plast Reconstr Aesthet Surg 2016;69:1211-7.

12. Wixtrom RN, Stutman RL, Burke RM, Mahoney AK, Codner MA.
Risk of breast implant bacterial contamination from endogenous breast flora, prevention with nipple shields, and implications for biofilm formation. Aesthet Surg J 2012;32:956-63.

13. McGuire P, Reisman NR, Zins J, Murphy DK. Risk factor analysis for capsular contracture, malposition, and late seroma in subjects receiving natrelle style 410 form-stable silicone breast implants. Plast Reconstr Surg 2015;136:117-8.

14. Benito-Ruiz J, Manzano ML, Salvador-Miranda L. Five-year outcomes of breast augmentation with form-stable implants: periareolar vs transaxillary. Aesthet Surg J 2017;37:46-56.

15. Jacobson JM, Gatti ME, Schaffner AD, Hill LM, Spear SL. Effect of incision choice on outcomes in primary breast augmentation. Aesthet Surg J 2012;32:456-62.

16. Hammond DC, Migliori MM, Caplin DA, Garcia ME, Phillips CA. Mentor contour profile gel implants: clinical outcomes at 6 years. Plast Reconstr Surg 2012;129:1381-91.

17. Maxwell GP, Van Natta BW, Bengtson BP, Murphy DK. Ten-year results from the natrelle 410 anatomical form-stable silicone breast implant core study. Aesthet Surg J 2015;35:145-55.

18. Asplund O, Gylbert L, Jurell G, Ward C. Textured or smooth implants for submuscular breast augmentation: a controlled study. Plast Reconstr Surg 1996;97:1200-6. 